\title{
Utilização de fitoterápicos por idosos: resultados de um inquérito domiciliar em Belo Horizonte (MG), Brasil
}

\author{
Lucianno D. P. Marliére, ${ }^{1}$ Andréia Q. Ribeiro, ${ }^{1}$ Maria das Graças L. Brandão, ${ }^{2}$ Carlos $H$. \\ Klein, ${ }^{3}$ Francisco de Assis Acurcio ${ }^{*, 1}$
}
${ }^{1}$ Departamento de Farmácia Social, Faculdade de Farmácia, Universidade Federal de Minas Gerais, Av. Antônio Carlos, 6627, Pampulha, 31270-901 Belo Horizonte-MG, Brasil,
${ }^{2}$ Departamento de Produtos Farmacêuticos, Faculdade de Farmácia, Universidade Federal de Minas Gerais, Av. Antônio Carlos, 6627, Pampulha, 31270-901 Belo Horizonte-MG, Brasil,
${ }^{3}$ Departamento de Epidemiologia e Métodos Quantitativos, Escola Nacional de Saúde Pública Sérgio Arouca, FIOCRUZ, Rua Leopoldo Bulhões, 1480, 21041-210 Rio de Janeiro-RJ, Brasil

\begin{abstract}
RESUMO: Os fitoterápicos constituem uma modalidade de terapia complementar ou alternativa diante das necessidades de saúde e seu uso tem sido crescente na população idosa de diversos países. Entretanto, apresentam interações medicamentosas e reações adversas importantes e sua utilização não deve ser indiscriminada. Este estudo objetivou caracterizar o perfil de utilização de fitoterápicos por aposentados e pensionistas do INSS, com 60 anos e mais, residentes em Belo Horizonte, MG. A partir do cadastro do INSS, selecionou-se uma amostra aleatória de 881 idosos para entrevista. Investigou-se a prevalência de uso de fitoterápicos e interações medicamentosas potenciais. Um total de 667 (80,3\%) dos selecionados foi entrevistado. Setenta e um participantes (10,6\%) utilizaram fitoterápicos nos últimos 15 dias, principalmente aqueles preparados a partir de extratos de ginkgo (41,8\%), aesculus (12,3\%) e isoflavonas de soja (8,2\%). Mais de 60\% dos fitoterápicos foram adquiridos em farmácias de manipulação. Aproximadamente 45\% dos usuários de fitoterápicos estavam expostos a pelo menos uma interação medicamentosa potencial entre eles e medicamentos sintéticos, tais como entre ginkgo e diuréticos tiazídicos (14) e antiagregante plaquetário/anticoagulantes (8). São necessárias estratégias de orientação para o uso racional de fitoterápicos entre idosos, mais vulneráveis aos prejuízos decorrentes da utilização inadequada desses.
\end{abstract}

Unitermos: Idosos, fitoterápicos, utilização de medicamentos, interações medicamentosas.

\begin{abstract}
Herbal drug use by elderly people: results from a domiciliary survey in Belo Horizonte (MG), Brazil”. The herbal drugs constitute an alternative or complementary therapy for the health needs and its use has been growing in the aged population of many countries. However, herbal drugs present important adverse effects and drug interactions and should not be indiscriminately used. The aim of this study was to investigate the profile of herbal drug use by elderly and pensioner residents of Belo Horizonte, Brazil. Eight hundred and eighty-one retirees were randomly selected from the Instituto Nacional do Seguro Social (INSS) database for in-home interview. The prevalence of herbal drugs use, and possible drug interactions were investigated. Results showed that 667 (80.3\%) of selected individuals were interviewed. Seventyone subjects $(10.6 \%)$ used herbal drugs in the past 15 days, mainly those prepared from ginkgo (41.8\%), horse chestnut (12.3\%), and soya extract (8.2\%). More than $60 \%$ of the herbal drugs were acquired in compounding pharmacies. Almost $45 \%$ of the herbal drug users were exposed to at least one potential herb-drug interaction, such as between ginkgo and thiazide diuretics (14) and antithrombotic/anticoagulants (8). Strategies to provide orientation for the rational use of these products in elderly people are necessary, since they are the population most exposed to polypharmacy and in consequence, most vulnerable to inappropriate uses of medications.
\end{abstract}

INTRODUÇÃO

Keywords: Elderly, herbal drugs, drug utilization, drug-herb interactions.

O Brasil tem vivenciado um processo de envelhecimento demográfico com particularidades que o destacam na escala mundial. Em 2005, o número de pessoas de 60 anos ou mais ultrapassou 18 milhões, correspondendo a quase $10 \%$ da população brasileira (IBGE, 2006).

A saúde é um setor bastante influenciado pelo envelhecimento e pelo aumento da longevidade. Nesse contexto, admite-se que o uso de medicamentos 
constitui uma intervenção importante para a recuperação e manutenção da saúde de grande parcela dos idosos.

Os fitoterápicos são considerados uma modalidade de terapia complementar ou alternativa em saúde e seu uso tem sido crescente (Carvalho et al., 2008; Veiga-Junior, 2008; Silva et al., 2006; Brownie, 2006; Tindle et al., 2005; Canter \& Ernst, 2004). Inquéritos de saúde realizados em vários países têm focalizado o uso desses medicamentos entre os idosos, sendo que alguns apontam uma maior utilização desses em comparação aos adultos jovens (Bardia et al., 2007; Canter \& Ernst, 2004) e que os principais fitoterápicos utilizados por idosos são ginkgo, equinacea e ginseng (Brownie, 2006; Bruno \& Ellis, 2005; Gunther et al., 2004).

Uma preocupação com os fitoterápicos advém do fato de que seu uso é, muitas vezes, associado ao conceito de inocuidade, de forma que se não fazem bem, não farão mal (Fonseca, 2008). Conseqüentemente, vários autores alertam que há grande probabilidade de que parte considerável de usuários faça uso dessa terapia sem, entretanto, informar aos prescritores ou outros profissionais de saúde (Brownie, 2006; Bruno \& Ellis, 2005; Canter \& Ernst, 2004; Dergal et al., 2002). Esse fato é de especial importância para os idosos que, em geral, utilizam elevado número de medicamentos e por período prolongado, o que os torna mais vulneráveis aos riscos associados à polifarmácia, tais como o maior potencial de interações medicamentosas (Alexandre et al., 2008a,b; Bruno \& Ellis, 2005).

No Brasil, poucos estudos têm focalizado os fitoterápicos no âmbito dos estudos farmacoepidemiológicos (Brandão et al., 2006; Ribeiro et al., 2005), e são desconhecidas pesquisas sobre essa temática na população idosa.

O presente estudo é conseqüência de uma iniciativa da Confederação Brasileira de Aposentados e Pensionistas (COBAP), a qual manifestou ao Ministério da Saúde interesse em colaborar com uma pesquisa sobre o perfil de consumo de medicamentos por idosos, a fim de que os resultados pudessem servir de subsídios para o aperfeiçoamento da política nacional de medicamentos. O objetivo principal da pesquisa foi descrever o perfil de utilização de medicamentos por aposentados e pensionistas do Instituto Nacional do Seguro Social (INSS), com 60 anos ou mais de idade, em três âmbitos: o Brasil e os municípios de Belo Horizonte e do Rio de Janeiro.

Neste artigo, apresenta-se a prevalência de uso de fitoterápicos entre os idosos participantes do inquérito domiciliar realizado em Belo Horizonte (MG), com ênfase nas interações medicamentosas potenciais observadas.

\section{MATERIAL E MÉTODOS}

O estudo possui delineamento seccional, com abordagem domiciliar. Foi aprovado pelo Comitê de
Ética em Pesquisa da UFMG.

A população alvo foi constituída por aposentados e/ou pensionistas, com idade de 60 anos ou mais, vinculados ao Regime Geral de Previdência Social do INSS/MPAS e residentes em Belo Horizonte (MG), no ano de 2002.

A seleção dos participantes foi realizada pela DATAPREV, a partir de seu cadastro de beneficiários, por amostragem aleatória simples. O tamanho da amostra foi definido considerando-se erro do tipo I de 0,05 , taxa de resposta de $75,0 \%$ e erro amostral entre $4 \%$ e $0,8 \%$ para prevalências entre $50 \%$ e $1 \%$, respectivamente. Inicialmente foram sorteados 800 indivíduos. Entretanto, para obter quantidades de entrevistas próximas ao planejado foi necessário sorteio adicional, em função da ocorrência de perdas. Este procedimento resultou em acréscimo de 81 indivíduos à amostra inicial. Detalhes sobre a metodologia estão descritos em Acurcio et al. (2006).

As informações foram obtidas por meio de questionário semi-estruturado com perguntas referentes a características sociodemográficas, condições de saúde, uso de serviços de saúde e uso de medicamentos nos 15 dias anteriores à realização da entrevista. Para evitar vieses, o período recordatório para essa informação foi de 15 dias e a grande maioria dos medicamentos citados teve seu uso comprovado por bulas e/ou embalagens e/ ou prescrições apresentadas pelos entrevistados.

Sobre cada uma das especialidades farmacêuticas utilizadas, identificou-se o fabricante, a forma farmacêutica, a duração do uso, a origem da prescrição/indicação e o local de obtenção. Remédios feitos em casa, como chás, ervas, raízes e remédios homeopáticos não foram investigados.

As entrevistas foram realizadas no período de março a junho de 2003, preferencialmente, com os indivíduos selecionados. Em situações de impedimento por motivos de saúde, tais como surdez ou déficit cognitivo, as entrevistas foram feitas com parentes ou cuidadores.

A classificação de fitoterápicos adotada nesse estudo obedece à legislação em vigor no Brasil, a qual estabelece que fitoterápico é aquele medicamento obtido empregando-se exclusivamente matérias-primas vegetais. Não se considera medicamento fitoterápico aquele que, na sua composição, inclua substâncias ativas isoladas, de qualquer origem, nem as associações destas com extratos vegetais (Brasil, 2004a).

Estimou-se a prevalência do uso de fitoterápicos nos 15 dias anteriores à entrevista; a distribuição de freqüência dos fitoterápicos mais utilizados, bem como a caracterização do uso: medicamento industrializado ou manipulado, forma farmacêutica, tempo de uso, fonte de indicação e local de obtenção. Foram identificadas e descritas as interações medicamentosas potenciais envolvendo os fitoterápicos utilizados. Interações medicamentosas potenciais ocorrem quando 
Tabela 1. Distribuição dos fitoterápicos utilizados pelos aposentados do INSS, de 60 anos ou mais, Belo Horizonte (MG), Brasil, 2003.

\begin{tabular}{l|c}
\hline \multicolumn{1}{c|}{ Fitoterápico } & Freqüência - $\mathrm{n}(\%)$ \\
\hline Ginkgo (Ginkgo biloba) & $41(41,8)$ \\
Castanha da Índia (Aesculus hippocastanum) & $12(12,3)$ \\
Isoflavonas de soja (Glycine max)* & $8(8,2)$ \\
Maracujá (Passiflora incarnata) & $4(4,1)$ \\
Cratego (Crataegus oxyacantha) & $3(3,1)$ \\
Plantago (Plantago ovata) & $3(3,1)$ \\
Sene (Senna alexandrina) & $3(3,1)$ \\
Alcachofra (Cynara scolymus) & $2(2,0)$ \\
Alcaçuz (Glycyrrhiza glabra) & $2(2,0)$ \\
Arnica (Arnica montana) & $2(2,0)$ \\
Cassia fistula & $2(2,0)$ \\
Garra-do-diabo (Harpagophytum procumbens) & $2(2,0)$ \\
Ginseng (Panax ginseng) & $2(2,0)$ \\
Guaco (Mikania glomerata) & $2(2,0)$ \\
Hamamelis (Hamamelis virginiana) & $2(2,0)$ \\
Poligala (Polygala senega) & $2(2,0)$ \\
Unha-de-gato (Uncaria tomentosa) & $2(2,0)$ \\
Valeriana (Valeriana officinalis) & $1(1,0)$ \\
Cascara Sagrada (Rhamnus purshiana) & $1(1,0)$ \\
Espinheira-Santa (Maytenus ilicifolia) & $1(1,0)$ \\
Guaraná (Paullinia cupana) & $1(1,0)$ \\
\hline Total & $98(100,0)$ \\
\hline
\end{tabular}

*Mistura de isoflavonas.

dois ou mais medicamentos reagem entre si, o que pode tornar o medicamento menos efetivo, causar reações adversas inesperadas ou aumentar a ação de um medicamento específico (FDA, 2006). As fontes de informação utilizadas para avaliação das interações medicamentosas foram: Herb contraindications and drug interactions (publicação especializada em interações medicamentosas envolvendo fitoterápicos) (Brinker \& Stodart, 2001), as monografias de fitoterápicos e plantas medicinais da European Scientific Cooperative on Phytoterapy (ESCOP, 1999) e da Organização Mundial da Saúde (WHO, 2002; WHO, 1999). Além dessas fontes, realizou-se uma busca na base de dados Pubmed por artigos de revisão publicados a partir de 2000. Nesse caso, foi utilizado o unitermo "herb-drug interactions". Para a classificação da gravidade do resultado das interações adotou-se a proposta de Bachmann et al. (2006), a saber: (0) sem classificação, (1) menor: os efeitos são considerados toleráveis na maioria dos casos e a intervenção médica é desnecessária, (2) moderada: intervenção médica necessária para tratar os efeitos, mas esses não satisfazem os critérios da categoria maior, (3) maior: os efeitos podem acarretar morte, hospitalização, lesão permanente ou fracasso terapêutico.

\section{RESULTADOS}

Dentre os 667 idosos que participaram da pesquisa, 63,7\% eram do sexo feminino, com idade média de 72,4 anos (dp = 7,6; amplitude $=61$ a 102) e $54,7 \%$ tinham escolaridade superior ao curso primário completo. As morbidades mais freqüentemente referidas foram os problemas de visão $(78,6 \%)$, a pressão alta (61,2\%), a depressão (32,1\%), os problemas de audição $(31,0 \%)$ e a artrite/reumatismo (29,8\%). O uso de pelo menos um medicamento nos últimos 15 dias foi relatado por $90,1 \%$ dos participantes, que utilizaram, em média, 4,1 produtos $(\mathrm{dp}=3,0)$ nesse período. Ocorreu polifarmácia (uso de 5 ou mais medicamentos) entre $40,0 \%$ do total de idosos entrevistados. A prevalência de uso de fitoterápicos nos 15 dias anteriores à entrevista foi de 10,6\%. Nesse período foi utilizado um total de 86 produtos fitoterápicos manipulados ou industrializados, constituídos de extratos de uma única planta ou em associações, resultando em 98 preparações. Na Tabela 1 são apresentados, em ordem decrescente de freqüência, os fitoterápicos utilizados. Destacam-se Ginkgo biloba (41,8\%), Aesculus hippocastanum (12,3\%) e isoflavonas de soja (8,2\%). Entre os entrevistados, 59,3\% utilizavam o fitoterápico há mais de um ano.

A maioria dos fitoterápicos consumidos (64\%) era manipulado. A forma farmacêutica mais utilizada foi a cápsula ou comprimido contendo o extrato seco padronizado da planta (88,4\%). Cerca de $66 \%$ dos fitoterápicos utilizados foram recomendados ou prescritos em consultório médico e $24 \%$ foram indicados por amigos, parentes ou vizinhos. A obtenção dos fitoterápicos utilizados nos últimos 15 dias ocorreu, predominantemente, na farmácia comercial (88,4\%).

Cerca de 45\% (32/71) dos idosos que relataram uso de fitoterápicos estavam expostos a pelo menos uma interação medicamentosa potencial entre fitoterápicos e medicamentos sintéticos. Dentre esses, quatro estavam sujeitos a duas interações potenciais envolvendo um 


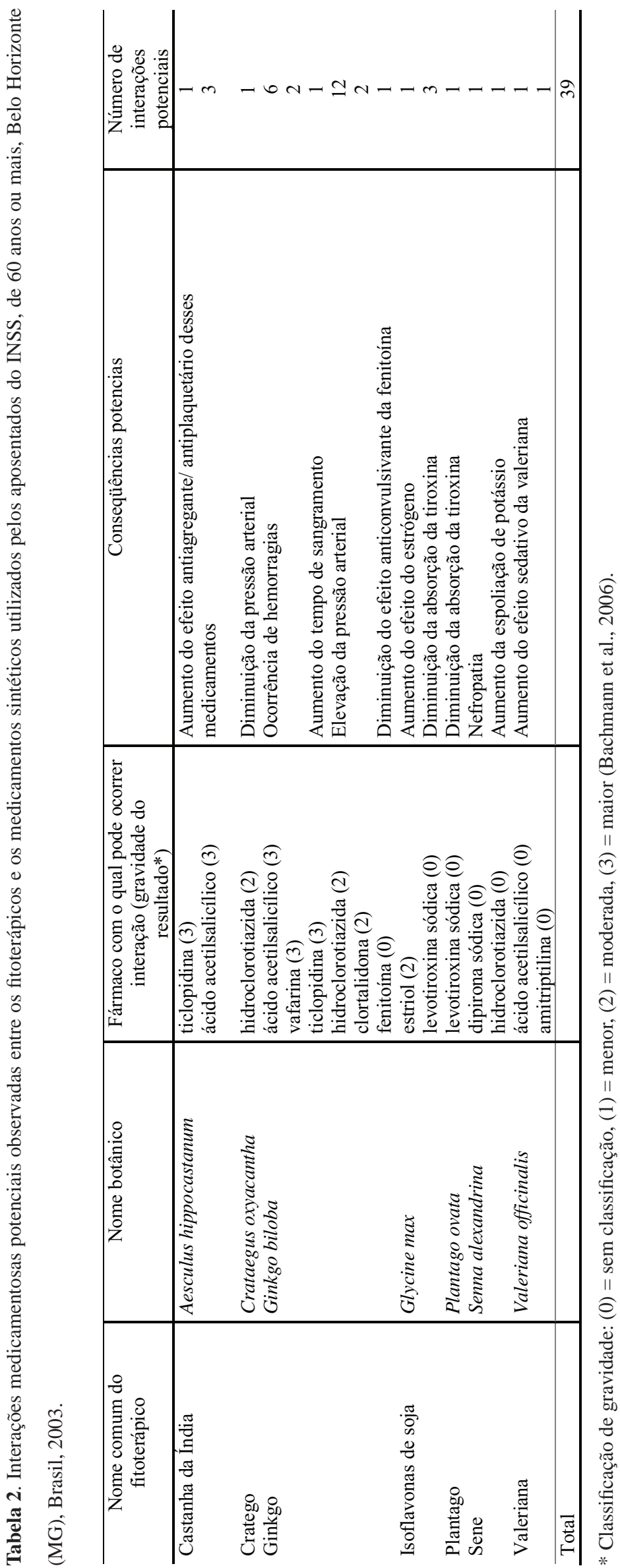


mesmo fitoterápico. Foram observadas 39 interações medicamentosas potenciais, envolvendo 32 fitoterápicos (Tabela 2). As principais interações medicamentosas potenciais ocorreram entre Ginkgo biloba e diuréticos tiazídicos (14) e entre o primeiro e antiagregante plaquetário/anticoagulantes (8). Tais interações são de gravidade moderada ou maior.

Entre os usuários de fitoterápicos, observouse uma proporção elevada de polifarmácia $(70,4 \%)$, comparada à proporção de $40,9 \%$ entre aqueles que não relataram uso de fitoterápicos.

\section{DISCUSSÃO}

Este estudo busca conhecer aspectos relacionados ao uso de fitoterápicos entre idosos brasileiros. Embora a extrapolação dos resultados deva ser feita com restrições, não é razoável supor que o perfil de utilização de fitoterápicos observado apresente divergências importantes em relação ao perfil de uso entre idosos brasileiros residentes em cidades mais populosas.

A prevalência de uso de fitoterápicos foi inferior à encontrada em outros países. Resultados do National Health Interview Survey (NHIS) revelaram que $12,9 \%$ dos idosos norte-americanos utilizaram ao menos um fitoterápico nos últimos 12 meses (Bruno \& Ellis, 2005). Maiores prevalências, em relação ao nosso inquérito, também foram registradas no Canadá (46,0\% e $17,0 \%$ ) e na Inglaterra (15,0\%) (Canter \& Ernst, 2004; McKenzie \& Keller, 2001). As diferenças nas estimativas podem ser parcialmente atribuídas a diferenças nos processos de regulação sanitária desses produtos. Nos países tomados como comparação, os fitoterápicos são considerados suplemento alimentar, de forma que podem ser consumidos sem prescrição médica. Logo, a maior utilização pelos idosos desses países pode ser devida ao acesso facilitado a tais produtos (Shahrokh et al., 2005; Canter \& Ernst, 2004; McKenzie \& Keller, 2001). No Brasil, fitoterápicos são considerados medicamentos pelos órgãos sanitários (BRASIL, 2004a). Ademais, alguns fitoterápicos utilizados pelos idosos do presente estudo, necessitam de prescrição médica para sua aquisição. Isso ocorre, por exemplo, com ginkgo, hamamelis e valeriana (BRASIL, 2004b).

Três fitoterápicos - ginkgo, castanha da índia e isoflavonas de soja responderam por mais da metade dos produtos utilizados, com grande discrepância do primeiro em relação aos demais. A maior freqüência de uso de Gingko biloba corrobora os achados de estudos conduzidos em outros países com a população idosa (Brownie, 2006; Shahrokh et al., 2005; Dergal et al., 2002). Em parte, pode-se justificar o maior uso desse fitoterápico à existência de um grande número de estudos científicos realizados com extratos padronizados dessa planta, que vem ganhando cada vez mais espaço na terapêutica (Suzuki, 2002). Por outro lado, é importante ressaltar que algumas das indicações terapêuticas apontadas para esse fitoterápico não dispõem de evidências científicas fortes, tais como sua eficácia no tratamento da demência e declínio cognitivo (Birks \& Grimley, 2007) inclusive em estudos específicos na população idosa (Carlson et al., 2007). Esse fato é um alerta para o uso indiscriminado de Ginkgo biloba por idosos. No que se refere às preparações com castanha da índia, sua principal indicação é para o tratamento de insuficiência venosa crônica (tais como varizes e hemorróidas). Vários estudos têm demonstrado a eficácia desse fitoterápico para essa indicação terapêutica na população adulta (Pitler \& Ernst, 2006; Siebert et al., 2002).

A freqüência observada de utilização de preparações com isoflavona de soja é, provavelmente, reflexo da queda no uso da terapia de reposição hormonal (TRH), fato que teve início a partir de meados do ano 2002. A maior atenção aos fitoestrógenos como alternativa no tratamento dos sintomas relacionados ao climatério se deu, principalmente, após a publicação de resultados de ensaios clínicos associando o uso da TRH com estrógenos isolados ou em combinação com progestógenos ao aumento da incidência de câncer de mama, acidente vascular cerebral, enfermidade tromboembólica, entre outros (Una recopilación, 2003). Entretanto, o uso racional do extrato de soja (mistura de isoflavonas) ainda carece de evidências acerca da sua eficácia na redução dos sintomas da menopausa (Cheng et al., 2007; Lethaby et al., 2007; Cassidi et al., 2006) e, mais ainda, na prevenção do câncer de mama (Rice \& Whitehead, 2006).

A maior parte dos fitoterápicos utilizados foi oriunda de farmácias de manipulação (64,0\%). Esta situação é preocupante, uma vez que nesses estabelecimentos podem ocorrer problemas como falta de controle de qualidade das matérias-primas manipuladas. Martins \& Brandão (2006) demonstraram, por exemplo, baixa qualidade de amostras manipuladas de castanhada-índia comercializadas na cidade de Belo Horizonte (MG). O consumo de medicamentos manipulados pode representar, assim, um perigo potencial para essa população idosa que geralmente convive com um grande número de doenças e utiliza vários medicamentos. Esse elevado consumo de medicamentos fitoterápicos manipulados que, em geral, são oferecidos a menor preço em comparação aos medicamentos sintéticos, pode ser justificado pelo baixo poder aquisitivo dessa população, pelo custo elevado dos fitoterápicos industrializados (cuja matéria prima é importada) e também pela pouca ou inexistente cobertura dos planos de saúde em relação a esses medicamentos. À luz desses resultados, é pertinente se discutir a inserção desses medicamentos na assistência farmacêutica básica no país, com vistas a garantir o acesso aos mesmos e o seu uso racional, com ênfase na segurança e eficácia. Nesse sentido, é fundamental a efetiva implantação da Política Nacional 
de Plantas Medicinais e Fitoterápicos (Brasil, 2006).

A principal fonte de indicação dos fitoterápicos usados pelos idosos foi a consulta médica. Embora no Brasil os fitoterápicos também sejam divulgados na mídia, o fato de serem considerados medicamentos e não suplementos pode explicar, em parte, esse resultado. Pode sinalizar, ainda, para um aumento do interesse e conhecimento referente aos fitoterápicos por parte do profissional médico, o que resulta em maior acesso a esses medicamentos via consultórios.

A freqüência de idosos expostos a pelo menos uma interação medicamentosa potencial observada acompanha os resultados de outros estudos internacionais, em que essa se situa entre $33 \%$ e $50 \%$ (Brownie, 2006; Canter \& Ernst, 2004; Dergal et al., 2002). Esse resultado indica baixa qualidade da prescrição de fitoterápicos para os idosos estudados, o que se agrava pelas considerações que se seguem. As principais possibilidades de interações envolveram medicamentos sintéticos de uso crônico, tais como diuréticos, antiagregantes plaquetários/anticoagulantes, significando uma exposição prolongada e, muitas vezes, desnecessária, ao risco de reações adversas graves como hemorragias e crises hipertensivas. Acrescente-se o fato de que a maioria dos usuários de fitoterápicos relatou esse uso por período superior a um ano. É possível, ainda, que nosso estudo tenha subestimado a real prevalência de interações medicamentosas potenciais, visto que muitos dos fitoterápicos ainda usados carecem de estudos clínicos bem conduzidos, em que as interações medicamentosas sejam contempladas.

Nossos resultados mostram que os idosos usuários de fitoterápicos utilizaram muitos e diferentes medicamentos concomitantemente, semelhante ao observado em outros países (Canter \& Ernst, 2004; Dergal et al., 2002; McKenzie \& Keller, 2001). Esta situação pode ter contribuído para a elevada freqüência de possibilidades de interações medicamentosas observadas, além de redundâncias, o que fala em favor de uma polifarmácia inadequada entre os idosos aposentados.

O conjunto desses resultados chama a atenção para algumas considerações. Pesquisas clínicas e farmacoepidemiológicas podem contribuir para garantir maior eficácia, efetividade e segurança dos medicamentos fitoterápicos, uma vez que elas contribuem na orientação do seu uso e na compreensão dos fatores a ele associados, inclusive e particularmente entre a população idosa, mais vulnerável à utilização inadequada de medicamentos. O estudo demonstra, ainda, a necessidade de ações educativas que aprimorem a prescrição e o uso desses produtos entre idosos no País. Uma maior divulgação e acesso à informação sobre esses medicamentos entre os prescritores e demais profissionais da área da saúde pode ser uma estratégia importante.

\section{REFERÊNCIAS}

Acurcio FA, Rozenfeld S, Ribeiro AQ, Klein CH, Moura CS, Andrade CR 2006. Utilização de medicamentos por aposentados brasileiros - I Metodologia e resultados de cobertura de inquérito multicêntrico. Cad Saúde Pública 22: 87-96.

Alexandre RF, Bagatini F, Simões CMO 2008a. Interações entre fármacos e medicamentos fitoterápicos à base de ginkgo ou ginseng. Rev Bras Farmacogn 18: 117126.

Alexandre RF, Bagatini F, Simões CMO 2008b. Potenciais interações entre fármacos e produtos à base de valeriana ou alho. Rev Bras Farmacogn 18: 455463.

Bachmann KA, Lewis JD, Fuller MA, Bonfiglio MF 2006. Interações medicamentosas. $2^{\mathrm{a}}$. ed. Barueri,SP: Manole, 887p.

Bardia A, Nisly NL, Zimmerman MB, Gryzlak BM, Wallace RB 2007. Use of herbs among adults based on evidence-based indications: findings from the National Health Interview Survey. Mayo Clin Proc 82: 561-566.

Birks J, Grimley EJ 2007. Ginkgo biloba for cognitive impairment and dementia. Cochrane Database Syst Rev 18: CD003120.

Brandão MGL, Acurcio FA, Montemor RLM, Marlière LDP 2006. Complementary/alternative medicine in Latin America: use of herbal remedies among a Brazilian metropolitan area population. $J$ Complement Integrative Medicine 3: 5.01-5.12.

Brasil 2004a. Ministério da Saúde. Agência Nacional de Vigilância Sanitária. RDC n 48 de 16 de março de 2004. Dispõe sobre o registro de medicamentos fitoterápicos. Diário Oficial, Brasília, 18 de março de 2004.

Brasil 2004b. Ministério da Saúde. Agência Nacional de Vigilância Sanitária. RE n 89 de 16 de março de 2004. Determina a publicação da Lista de registro simplificado de fitoterápicos. Diário Oficial, Brasília, 18 de março de 2004.

Brasil 2006. Ministério da Saúde. Decreto no 5.813, de 22 de junho de 2006. Aprova a Política Nacional de Plantas Medicinais e Fitoterápicos e dá outras providências. Disponível em: <http://portal.saude.gov.br/portal/ arquivos/pdf/Decreto_Fito.pdf>. Acesso em: $24 \mathrm{fev}$. 2008.

Brinker F, Stodart N 2001. Herb contraindications and drug interactions. $3^{\text {rd }}$ ed. Sandy, Oregon: Eclectic Medical Publications, 440p.

Brownie S 2006. Predictors of dietary and health supplement use in older Australians. Aust J Adv Nurs 23: 26-31.

Bruno JJ, Ellis JJ 2005. Herbal use among US elderly: 2002 National Health Interview Survey. Ann Pharmacother 39: 643-648.

Canter PH, Ernst E 2004. Herbal supplement use by persons aged over 50 years in Britain. Drugs Aging 21: 597605.

Carlson JJ, Farquhar JW, Dinucci E, Ausserer L, Zehnder J, Miller D, Berra K, Hagerty L, Haskell WL 2007. Safety and efficacy of a Ginkgo biloba-containing dietary supplement on cognitive function, quality of life, and platelet function in healthy, cognitively 
intact older adults. J Am Diet Assoc 107: 422-432.

Carvalho ACB, Balbino EE, Maciel A, Perfeito JPS 2008. Situação do registro de medicamentos fitoterápicos no Brasil. Rev Bras Farmacogn 18: 314-319.

Cassidy A, Albertazzi P, Lise-Nielsen I, Hall W, Williamson G, Tetens I, Atkins S, Cross H, Manios Y, Wolk A, Steiner C, Branca F 2006. Critical review of health effects of soyabean phyto-oestrogens in postmenopausal women. Proc Nutr Soc 65: 76-92.

Cheng G, Wilczek B, Warner M, Gustafsson JA, Landgren BM 2007. Isoflavone treatment for acute menopausal symptoms. Menopause 14: 468-473.

Dergal JM, Gold JL, Laxer DA, Lee MSW, Binns MA, Lanctot KL, Freedman M, Rochon PA 2002. Potential interactions between herbal medicines and conventional drug therapies used by older adults attending a memory clinic. Drugs Aging 19: 879886.

ESCOP 1999. European Scientific Cooperative on Phytotherapy. Monographs on the medicinal uses of plants drugs. United Kingdom: ESCOP.

FDA 2006. Food and Drug Administration. Drug interactions. Disponível em: <http://www.fda.gov/cder/ consumerinfo/druginteractions.htm> Acesso em: 28 abr. 2006.

Fonseca AL 2008. Medicamentos fitoterápicos. In: Fonseca, A.L. Interações medicamentosas. $4^{\mathrm{a}}$. ed. São Paulo: EPUB, p. 531-534.

Gunther S, Patterson RE, Kristal AR, Stratton KL, White E 2004. Demographic and health-related correlates of herbal and specialty supplement use. J Am Diet Assoc 104: 27-34.

IBGE 2006. Instituto Brasileiro de Geografia E Estatística. Síntese de indicadores sociais 2006. Rio de Janeiro: 2006. 317p. (Estudos \& Pesquisas - Informação demográfica e socioeconômica, 19) Disponível em: <http://www.ibge.gov.br>. Acesso em: 25 mai. 2007.

Lethaby AE, Brown J, Marjoribanks J, Kronenberg F, Roberts H, Eden J 2007. Phytoestrogens for vasomotor menopausal symptoms. Cochrane Database Syst Rev 17: CD001395.

Martins EMLP, Brandão MGL 2006. Qualidade de amostras comerciais preparadas com Aesculus hippocastanum L. (Castanha-da-Índia). Rev Bras Farmacogn 16: 224-229.

McKenzie J, Keller HH2001. Vitamin-mineral supplementation and use of herbal preparations among communityliving older adults. Can J Public Health 92: 286290.

Pittler MH, Ernst E 2006. Horse chestnut seed extract for chronic venous insufficiency. Cochrane Database Syst Rev 25: CD003230.

Ribeiro AQ, Leite JPV, Dantas-Barros AM 2005. Perfil de utilização de fitoterápicos em farmácias comunitárias de Belo Horizonte sob a influência da legislação nacional. Rev Bras Farmacogn 15: 65-70.

Rice S, Whitehead SA 2006. Phytoestrogens and breast cancerpromoters or protectors? Endocr Relat Cancer 13: 995-1015.

Shahrokh LE, Lukaszuk JM, Prawitz AD 2005. Elderly herbal supplement users less satisfied with medical care than nonusers. J Am Diet Assoc 105: 1138-1140.
Siebert U, Brach M, Sroczynski G, Berla K 2002 Efficacy, routine effectiveness, and safety of horsechestnut seed extract in the treatment of chronic venous insufficiency. A meta-analysis of randomized controlled trials and large observational studies. Int Angiol 21: 305-315.

Silva MIG, Gondim APS, Nunes IFS, Sousa FCF 2006. Utilização de fitoterápicos nas unidades básicas de atenção à saúde da família no município de Maracanaú (CE). Rev Bras Farmacogn 16: 455-462.

Suzuki SF 2002. O mercado de medicamentos fitoterápicos no Brasil. In: Schulz V, Hansel R, Tyler VE. Fitoterapia racional. 1ed. Barueri: Manole, p. 363-369.

Tindle HA, Davis RB, Phillips RS, Eisenberg DM 2005. Trends in use of complementary and alternative medicine by US adults: 1997-2002. Altern Ther Health Med 11: 42-49.

Una recopilación de novedades 2003 en terapéutica. Butlletí Groc 16: 7.

Veiga-Junior VF 2008. Estudo do consumo de plantas medicinais na Região Centro-Norte do Estado do Rio de Janeiro: aceitação pelos profissionais de saúde e modo de uso pela população. Rev Bras Farmacogn 18: 308-313.

WHO 1999. World Health Organization. Monographs on selected medicinal plants. Volume 1. Genebra: World Health Organization, 294 p.

WHO 2002. World Health Organization. Monographs on selected medicinal plants. Volume 2. Genebra: World Health Organization, 362 p. 